\title{
Spin-orbit coupling, edge states and quantum spin Hall criticality due to Dirac fermion confinement: the case study of graphene
}

\author{
G. Tkachov ${ }^{\mathrm{a}}$ and M. Hentschel \\ Max-Planck Institute for the Physics of Complex Systems, Dresden, Germany
}

Received 23 October 2008 / Received in final form 28 April 2009

Published online 3 June 2009 - (c) EDP Sciences, Società Italiana di Fisica, Springer-Verlag 2009

\begin{abstract}
We propose a generalized Dirac fermion description for the electronic state of graphene terminated by a zigzag edge. This description admits a specific spin-orbit coupling needed to preserve timereversal invariance of the zigzag confinement, otherwise, for spinless particles, showing the parity anomaly typical of quantum electrodynamics in $(2+1)$ dimensions. At a certain critical strength the spin-orbit coupling induces a phase transition of the quantum-spin-Hall type. It is manifested by a novel type of the edge states consisting of a Kramers' pair of counter-propagating modes with opposite spin orientations. Such edge states are capable of accumulating an integer spin in response to a transverse electric field in the absence of a magnetic one. They exist without any excitation gap in the bulk, due to which our system stands out among other quantum spin Hall systems studied earlier. We show that at the transition the local density of states is discontinuous and its energy dependence reflects the phase diagram of the system.
\end{abstract}

PACS. 73.20.At Surface states, band structure, electron density of states - 73.23.-b Electronic transport in mesoscopic systems

\section{Introduction}

Massless Dirac fermions in graphene, an isolated two dimensional (2D) graphite layer, are responsible for unconventional electronic properties of this material [1,2], offering new functionalities for nanoelectronic devices such as recently realized single-electron transistors in graphene quantum dots [3]. This is a typical example of a situation where Dirac fermions occur in a confined geometry, which brings up the rather general issue of the boundary effects in graphene. The need for their characterization is one of the outstanding current challenges in the field, closely related to the problem of Klein tunneling, and, for this reason, having no analogues in conventional semiconductors and metals.

In the present paper we consider a novel spin-orbit coupling mechanism originating entirely from Dirac fermion confinement rather than being an intrinsic material property. As a model, we consider graphene bounded by a zigzag edge and without spin-orbit coupling in the bulk. The zigzag boundary is one of the most common types of the honeycomb lattice termination (shown in Fig. 1) that stands out due to its unique ability to support edge states, decaying in the bulk and delocalized along the boundary [4-17]. The existence of the edge states is crucial for the spin-orbit coupling in our model. To demonstrate this, we first argue that for spinless electrons the zigzag confinement exhibits an instability toward the formation of

\footnotetext{
a e-mail: tkachov@pks.mpg.de
}

chiral edge states. This would break time-reversal symmetry and drive the system into a state analogous to the integer quantum Hall phase proposed by Haldane [18] as a realization of the parity anomaly of $(2+1)$-dimensional quantum electrodynamics (e.g. Ref. [19]).

We further show that for spin-half particles the requirement for the cancellation of the parity anomaly implies the coupling between the spin and orbital degrees of freedom: Instead of the chiral edge we obtain a pair of counter-propagating gapless edge modes with the opposite spin orientations. They are related to each other by time-reversal symmetry and have a Kramers' degenerate spectrum, ensuring their robustness against time-reversal invariant perturbations.

Such a Kramers' doublet of gapless edge states is a characteristic signature of the quantum spin Hall (QSH) systems (e.g. Refs. [20-23]). The recent interest in these systems is motivated by the principal possibility to realize a time-reversal invariant integer quantum Hall state in which the spin Hall conductance is quantized. The quantization is due to the spin accumulation ability of the edge states that does not require a strong magnetic field. The transition into the QSH state discussed in the literature [20-23] is accompanied by opening a finite excitation gap in the $2 \mathrm{D}$ bulk generated, e.g. by spin-orbit interactions. In this respect, our case is special since zigzagterminated graphene (as well as the unbounded system) is a zero-gap semiconductor, and the spin-orbit coupling at the edge does not influence the bulk electronic states. 


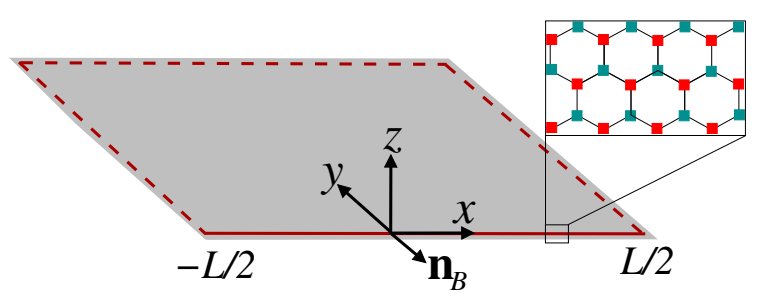

Fig. 1. Geometry: system occupies the region $|x| \leq L / 2, y \geq 0$ with a zigzag edge at $y=0$ described by the boundary condition, equation (1), and is periodic in $x$ direction. $\mathbf{n}_{B}$ is the boundary normal.

Nonetheless, we show that the system does exhibit a transition from the ordinary zero-gap semiconductor phase with spin-degenerate edge states $[4,16]$ to the novel phase possessing a Kramers' pair of gapless spin-filtered edge modes, which can be identified as a QSH state.

The absence of the bulk energy gap makes it difficult to characterize the QSH state by electric transport means. We suggest an alternative method based on the tunneling spectroscopy of the local density of states (LDOS). In the QSH and ordinary states, the LDOS shows drastically different energy dependences that cannot be continuously transformed into each other, implying that the two states are topologically distinct.

\section{Discrete symmetries of the problem}

We start with the spinless model containing a pair of Weyl fermions originating from the two inequivalent valleys of graphene's Brillouin zone [19]. They can be represented by a four-component function $\psi$ satisfying the Dirac equation $\epsilon \psi=-i \hbar v\left(\tau_{z} \otimes \boldsymbol{\Sigma}\right) \boldsymbol{\nabla} \psi$ with energy $\epsilon$ measured from the Fermi level and momentum $-i \hbar \nabla$ confined to the graphene plane, see Figure 1 ( $v$ is the Fermi velocity). We consider a single edge (along the $x$-axis) that does not cause inter-valley scattering, which can be described by an effective boundary condition of reference [24]:

$$
\begin{gathered}
\psi=\left.M \psi\right|_{y=0} \\
M=\cos \left(\Lambda \tau_{z}+\zeta \tau_{0}\right) \otimes \Sigma_{x}+\sin \left(\Lambda \tau_{z}+\zeta \tau_{0}\right) \otimes \Sigma_{z} .
\end{gathered}
$$

The Pauli matrices $\Sigma_{x, z}$ act in sublattice (pseudospin) space, while $\tau_{z}$ and the unit matrix $\tau_{0}$ operate in valley space. Equation (1) contains two parameters, $\zeta$ and $\Lambda$, that in the limit $\zeta \rightarrow 0, \Lambda \rightarrow \frac{\pi}{2}$ yield $M=\tau_{z} \otimes \Sigma_{z}$ which is the continuum model for a zigzag graphene edge $[9,16]$. We intend to study the stability of the zigzag edge states with respect to small deviations from the zigzag boundary condition. To introduce such deviations we use the general formula for $M(1)$, assuming small but finite parameters

$$
\zeta \ll 1, \quad \lambda=\Lambda-\pi / 2 \ll 1 .
$$

They explicitly violate $2 \mathrm{D}$ parity and inversion symmetries of the system, respectively, allowing us to study their interplay, which has not been done previously $[8,16,25]$.
The 2D parity of the Dirac equation is defined as coordinate reflection along the edge, $x \rightarrow-x$ accompanied by a unitary spinor transformation [19],

$$
\psi(x, y) \rightarrow \mathcal{P} \psi(-x, y), \quad \mathcal{P}=\tau_{x} \otimes \Sigma_{x}
$$

The $2 \mathrm{D}$ inversion is realized by an in-plane rotation by $\pi$, yielding $x, y \rightarrow-x,-y$, along with the spinor transformation

$$
\psi(x, y) \rightarrow \mathcal{I} \psi(-x,-y), \quad \mathcal{I}=\tau_{0} \otimes \Sigma_{z} .
$$

The boundary condition does not share these symmetries since both $M^{\mathcal{P}}=\mathcal{P} M \mathcal{P}^{\dagger}$ and $M^{\mathcal{I}}=\mathcal{I} M \mathcal{I}^{\dagger}$ differ from $M$ in equation (1):

$$
\begin{gathered}
M^{\mathcal{P}}=\cos \left(\Lambda \tau_{z}-\zeta \tau_{0}\right) \otimes \Sigma_{x}+\sin \left(\Lambda \tau_{z}-\zeta \tau_{0}\right) \otimes \Sigma_{z}, \\
M^{\mathcal{I}}=-\cos \left(\Lambda \tau_{z}+\zeta \tau_{0}\right) \otimes \Sigma_{x}+\sin \left(\Lambda \tau_{z}+\zeta \tau_{0}\right) \otimes \Sigma_{z} \cdot(6)
\end{gathered}
$$

Setting $\zeta \rightarrow 0$ restores the parity $\left(M^{\mathcal{P}} \rightarrow M\right)$, and if, additionally, $\lambda \rightarrow 0$ the inversion symmetry is recovered as well $\left(M^{\mathcal{I}} \rightarrow M\right)$.

We note that for $\zeta \neq 0$ the $2 \mathrm{D}$ parity is broken simultaneously with the time-reversal $(\mathcal{T})$ symmetry as the latter is represented by the operator $\mathcal{T}=\mathcal{P C}[16]$ where $\mathcal{C}$ is complex conjugation. Despite the violation of the $\mathcal{T}$ symmetry, the use of equation (1) is justified because $\mathcal{T}$ is only an effective symmetry of spinless particles (no spin degrees of freedom involved so far). Apart from that, the $\mathcal{T}$ symmetry breaking is considered as a weak perturbation with $\zeta \ll 1$. The small parameters $\zeta$ and $\lambda$ compete, and, therefore, it is natural to carry out the stability analysis as a two-parametric problem. This analysis will eventually lead us to the time-reversal invariant boundary condition (23), which is behind the main results of this study, e.g. the prediction of the quantum spin Hall transition.

\section{Spinless edge states and parity anomaly}

Let us first understand the implications of the broken symmetries for the equilibrium properties of spinless electrons which can be described by the LDOS,

$$
\mathcal{V}(\epsilon, \mathbf{r})=-(1 / \pi) \operatorname{Im} \operatorname{Tr} G^{R}(\mathbf{r}, \mathbf{r}),
$$

where the trace of the retarded matrix Green's function $G^{R}$ is taken in $\tau \otimes \Sigma$ space. $G^{R}$ satisfies the Dirac equation,

$$
\epsilon G^{R}\left(\mathbf{r}, \mathbf{r}^{\prime}\right)+i \hbar v\left(\tau_{z} \otimes \boldsymbol{\Sigma}\right) \nabla G^{R}\left(\mathbf{r}, \mathbf{r}^{\prime}\right)=\delta\left(\mathbf{r}-\mathbf{r}^{\prime}\right),
$$

the boundary condition (1), and $\left.G^{R}\right|_{y \rightarrow \infty}<\infty$. We also assume a periodic boundary condition in the $x$ direction (with period $L$ ), modulated by a magnetic phase $\phi$ :

$$
\left.G^{R}\right|_{x=L / 2}=\left.G^{R}\right|_{x=-L / 2} e^{2 \pi i \phi} .
$$

$G^{R}$ can be expanded in plane waves $e^{i k_{n} x}$ with

$$
k_{n}=\frac{2 \pi}{L}(n+\phi), \quad n \in Z(0, \pm 1, \ldots),
$$


following from equation (9). Then, the matrix Dirac equation can be reduced to ordinary differential equations for the diagonal elements of $G^{R}$ which can be readily solved (see, e.g. Ref. [17]). The final result is

$$
\begin{aligned}
& G^{R}\left(\mathbf{r}, \mathbf{r}^{\prime}\right)=\sum_{\tau= \pm 1, n \in Z}\left(\frac{\tau_{0}+\tau \tau_{z}}{2}\right) \otimes\left(\Sigma_{0}+\frac{\tau \hbar v}{i \epsilon} \boldsymbol{\Sigma} \nabla\right) \\
& \times\left(G_{\tau k_{n}}^{+}\left(y, y^{\prime}\right) \Sigma_{0}+G_{\tau k_{n}}^{-}\left(y, y^{\prime}\right) \Sigma_{z}\right) \frac{e^{i k_{n}\left(x-x^{\prime}\right)}}{L} \\
& G_{\tau k_{n}}^{+}\left(y, y^{\prime}\right)=\frac{\epsilon}{2 \hbar^{2} v^{2} q_{n}}\left(e^{-q_{n}\left(y+y^{\prime}\right)}-e^{-q_{n}\left|y-y^{\prime}\right|}\right) \\
& +\frac{q_{n}+k_{n} s_{\tau}}{2\left(\epsilon-\tau c_{\tau} \hbar v k_{n}\right)} e^{-q_{n}\left(y+y^{\prime}\right)} \\
& G_{\tau k_{n}}^{-}\left(y, y^{\prime}\right)=\frac{k_{n}+q_{n} s_{\tau}-\tau c_{\tau} \epsilon / \hbar v}{2\left(\epsilon-\tau c_{\tau} \hbar v k_{n}\right)} e^{-q_{n}\left(y+y^{\prime}\right)}
\end{aligned}
$$

Here $\tau= \pm 1$ labels the valleys and $q_{n}=\sqrt{k_{n}^{2}-\epsilon^{2} / \hbar^{2} v^{2}}$. The edge-state spectrum follows from equation (12) taken at $\epsilon \rightarrow \tau c_{\tau} \hbar v k_{n}$ :

$$
G_{\tau k_{n}}^{+}\left(y, y^{\prime}\right) \approx \frac{k_{n} s_{\tau} \Theta\left(k_{n} s_{\tau}\right)}{\epsilon-\tau c_{\tau} \hbar v k_{n}} e^{-k_{n} s_{\tau}\left(y+y^{\prime}\right)} .
$$

It has a pole only if the unit step function $\Theta\left(k_{n} s_{\tau}\right)$ is nonzero, which determines the spectrum as

$$
\epsilon_{\tau}\left(k_{n}\right)=\tau c_{\tau} \hbar v k_{n} \quad \text { for } \quad k_{n} s_{\tau}>0
$$

with $c_{\tau}=\cos (\Lambda \tau+\zeta)$ and $s_{\tau}=\sin (\Lambda \tau+\zeta)$. It is particlehole asymmetric due to the broken discrete symmetries.

Using equation (11) and the Poisson summation formula we can express the LDOS as

$$
\begin{aligned}
\mathcal{V}(\epsilon, y) & =-\frac{2}{\pi L} \sum_{\tau, n \in Z} \operatorname{Im} G_{\tau k_{n}}^{+}(y, y) \\
& =-\frac{1}{\pi^{2}} \sum_{\tau, n \in Z} e^{-2 \pi i n \phi} \int_{-\infty}^{\infty} d k e^{i n k L} \operatorname{Im} G_{\tau k}^{+}(y, y)
\end{aligned}
$$

where the integration over the edge $(|k| \geq|\epsilon| / \hbar v)$ and bulk $(|k| \leq|\epsilon| / \hbar v)$ states should be done separately. Accordingly, the $\operatorname{LDOS} \mathcal{V}(\epsilon, y)=\mathcal{V}_{e}(\epsilon, y)+\mathcal{V}_{b}(\epsilon, y)$ contains the edge contribution,

$$
\begin{aligned}
& \mathcal{V}_{e}(\epsilon, y)=-\sum_{\tau, n \in Z}\left|2 \pi \hbar v c_{\tau}\right|^{-1} \Theta\left(\epsilon \tau s_{\tau} c_{\tau}\right) \\
& \times \exp i n\left(\frac{\epsilon L}{\hbar v c_{\tau}}-2 \pi \phi\right) \partial_{y} \exp \left(-\frac{2 y}{\hbar v}\left|\frac{\epsilon s_{\tau}}{c_{\tau}}\right|\right),
\end{aligned}
$$

and the bulk one,

$$
\begin{aligned}
\mathcal{V}_{b}(\epsilon, y)= & \frac{|\epsilon|}{\pi^{2} \hbar^{2} v^{2}} \sum_{\tau, n \in Z} \int_{0}^{\pi / 2} d \gamma \\
& \times\left\{\operatorname { c o s } ( 2 \pi n \phi ) \operatorname { c o s } ( n k _ { \epsilon } L \operatorname { c o s } \gamma ) \left[1-\cos ^{2} \gamma\right.\right. \\
\times & \left.\frac{s_{\tau}^{2} \cos \left(\frac{2 y \epsilon}{\hbar v} \sin \gamma\right)+\tau s_{\tau} c_{\tau} \sin \gamma \sin \left(\frac{2 y \epsilon}{\hbar v} \sin \gamma\right)}{s_{\tau}^{2}+c_{\tau}^{2} \sin ^{2} \gamma}\right] \\
& \left.+\frac{\tau c_{\tau} \sin (2 \pi n \phi) \sin \left(n k_{\epsilon} L \cos \gamma\right) \sin \gamma \cos \gamma}{s_{\tau}^{2}+c_{\tau}^{2} \sin ^{2} \gamma} \sin \gamma\right)-s_{\tau} \sin \left(\frac{2 y \epsilon}{\hbar v} \sin \gamma\right)
\end{aligned}
$$

The edge LDOS (17) vanishes for $s_{\tau}=0$, i.e. for in-plane pseudospin orientation at the edge (see, Eq. (1)). This points to the topological origin of the edge states, since their existence requires a nontrivial 3D pseudospin structure with the out-of-plane component $\Sigma_{z}$.

If we now restore the symmetries, setting $\zeta, \lambda \rightarrow 0$ (i.e. $c_{\tau} \rightarrow 0$ and $s_{\tau} \rightarrow \tau$ ), the bulk LDOS (18) recovers the particle-hole symmetry, while the edge one, equation (17), fails to do so because of the singularity at $c_{\tau}=0$. This anomalous asymmetry leads to a finite charge density,

$$
\rho(y)=e \int_{-\infty}^{0} d \epsilon[\mathcal{V}(\epsilon, y)-\mathcal{V}(-\epsilon, y)] / 2,
$$

entirely localized at the edge, since the symmetric bulk LDOS cancels out. By analogy with reference [17] $\rho(y)$ can be calculated from equation (17) as

$$
\begin{aligned}
\rho(y) & =-\frac{e}{4 L} \partial_{y} \frac{\cosh \left[\frac{4 \pi y}{L}\left(\phi-\frac{\operatorname{sgn} \phi}{2}\right)\right] \sum_{\tau} \operatorname{sgn}(\lambda+\tau \zeta)}{\sinh (2 \pi y / L)} \\
& +\frac{e}{4 L} \partial_{y} \frac{\sinh \left[\frac{4 \pi y}{L}\left(\phi-\frac{\operatorname{sgn} \phi}{2}\right)\right] \sum_{\tau} \operatorname{sgn}(\lambda \tau+\zeta)}{\sinh (2 \pi y / L)},
\end{aligned}
$$

where the small parameters $\zeta$ and $\lambda$ enter through the sign function, and the dimensionless magnetic flux is confined to the interval $0<|\phi|<1$ due to periodicity of equation (9). The net edge charge, $Q=L \int_{0}^{\infty} d y \rho(y)$ contains a flux-dependent contribution given by

$$
Q(\phi)=N e\left(\phi-\frac{\operatorname{sgn} \phi}{2}\right), N=\frac{1}{2} \sum_{\tau= \pm 1} \operatorname{sgn}(\lambda \tau-\zeta)
$$

Remarkably, adiabatic sweeping of the flux from 0 to 1 leads to the accumulation of the integer charge $\Delta Q=$ $Q(1)-Q(0)=N e$ with $N=0, \pm 1$. The nontrivial integers $N= \pm 1$ require $|\zeta|>|\lambda|$. This is the condition for the formation of a chiral Dirac fermion edge channel, which follows from the edge-state spectrum, equation (15), linearized with respect to $\zeta$ and $\lambda$,

$$
\epsilon_{\tau}\left(k_{n}\right)=-(\lambda \tau+\zeta) \hbar v k_{n} \quad \text { for } \quad k_{n} \tau>0
$$

We see that for $|\zeta|>|\lambda|$, the two graphene valleys prodive a pair of Weyl fermion edge states propagating in the same direction, i.e. a single chiral Dirac fermion edge channel. 
The interpretation of equation (21) is quite straightforward if we notice that the periodicity of our system (due to Eq. (9) is that of a cylinder enclosing a magnetic flux $\phi$ (in units of $c h / e$ ). Repeating Laughlin's argument [26] one can identify $\Delta Q$ as the Hall charge accumulated in the chiral edge channel in response to the electric field $E_{x}=-(h / e L) \dot{\phi}$ generated by the adiabatically varying flux.

On the other hand, since there is no quantizing magnetic field, our result is close in spirit to Haldane's integer quantum Hall effect [18] realizing the so-called parity anomaly [19]. The anomaly consists in the odd $\phi$-dependence of $Q$, which is in sharp contrast to the common expectation that the electric charge (a scalar quantity) should be an even function of the magnetic flux. Equation (21) has nevertheless normal parity. Indeed, the parity operation, equation (3) is equivalent to $\zeta, \phi \rightarrow-\zeta,-\phi$ which does not affect $Q$. Thus, the $N= \pm 1$ quantum Hall phases are characterized by the number of the chiral modes $(|N|=1)$ and two possible propagation directions $( \pm)$.

The parity anomaly indicates a magnetic instability of the continuum zigzag-edge model with respect to the small $\mathcal{T}$ symmetry breaking perturbation. Importantly, for $\lambda=0$ it takes only an infinitesimally small perturbation $\zeta$ to drive the system into the quantum Hall state with $N=$ $\operatorname{sgn} \zeta$. The conventional zero-gap semiconductor state (i.e. $N=0)$ is recovered when $\zeta=0$ and $\lambda$ is finite. The latter could originate from a staggered boundary potential [16].

While the parity anomaly and its connection to the quantum Hall physics are of interest on their own grounds, in what follows we return to the $\mathcal{T}$ symmetric situation. As shown previously in reference [20], the $\mathcal{T}$ invariance can be recovered in the presence of two spin- $1 / 2$ fermion species experiencing opposite-sign quantum Hall effects. Since the spatial 2D parity remains broken, we expect a confinement-induced spin-orbit coupling with the strength controlled by $\zeta$. It could for instance be induced by applying mechanical strain at zigzag graphene edges. In this paper we do not calculate the spin-orbit coupling strength on the microscopic basis. Such a situation is not that uncommon in the theory of spin-orbit interacting systems [27]. Despite its limitations, our model contains a quantum spin Hall (QSH) transition, allowing us to investigate its manifestations in observables.

\section{Edge spin-orbit coupling and QSH transition}

Let us combine the pseudospinors for opposite spin projections $\uparrow$ and $\downarrow$ (e.g. on $z$ axis) into a single function $\Psi=\left(\psi_{\uparrow}, \psi_{\downarrow}\right)$ and consider the following generalization of the boundary condition, equation (1): $\Psi=\mathcal{M} \Psi$,

$$
\begin{aligned}
\mathcal{M}= & \cos \left(\Lambda \sigma_{0} \otimes \tau_{z}+\zeta \sigma_{z} \otimes \tau_{0}\right) \otimes \Sigma_{x} \\
& +\sin \left(\Lambda \sigma_{0} \otimes \tau_{z}+\zeta \sigma_{z} \otimes \tau_{0}\right) \otimes \Sigma_{z},
\end{aligned}
$$

where $\sigma_{0}, \sigma_{z}$ are unit and Pauli matrices in spin space. The presence of $\sigma_{z}$ makes the new boundary condition

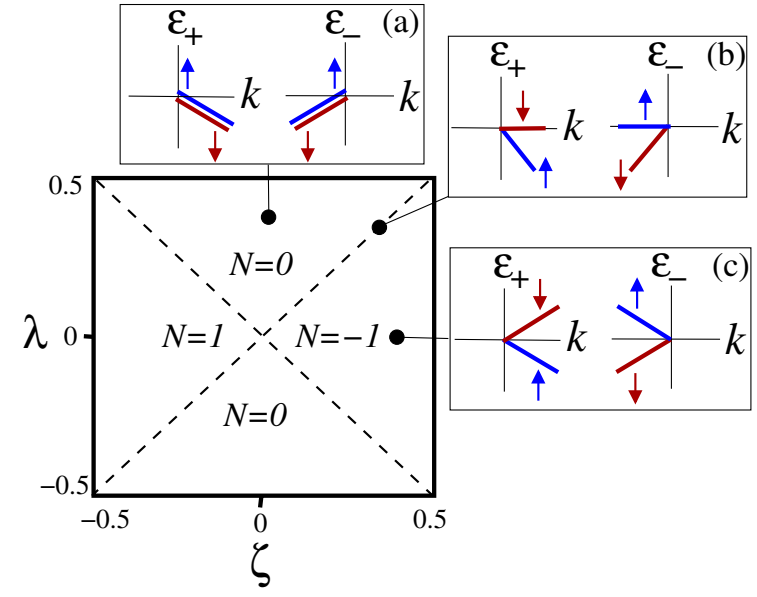

Fig. 2. Phase diagram in space of parameters $\zeta$ and $\lambda$ breaking 2D parity and inversion, respectively. $N=0$ and $N= \pm 1$ label ordinary zero-gap semiconductor and quantum spin Hall states, respectively. The critical point $\zeta=\lambda=0$ corresponds to the parity- and inversion-symmetric zigzag edge. Panels (a), (b) and (c) show the edge-state spectrum, equation (24), for $\tau= \pm 1$.

invariant under the time reversal operation $\mathcal{T}=i \sigma_{y} \otimes \tau_{x} \otimes$ $\Sigma_{x} \mathcal{C}$ where $\mathcal{C}$ is the complex conjugation. This also follows from the new edge spectrum obtained from equation (22) by $\zeta \rightarrow \zeta \sigma$ where $\sigma= \pm 1$ are the eigenvalues of $\sigma_{z}$ :

$$
\epsilon_{\tau \sigma}\left(k_{n}\right)=-(\lambda \tau+\zeta \sigma) \hbar v k_{n}, \quad \text { for } \quad k_{n} \tau>0 .
$$

In the presence of the spin-orbit term $(\propto \zeta)$ equation (24) exhibits Kramers' degeneracy $\epsilon_{-\tau,-\sigma}\left(-k_{n}\right)=\epsilon_{\tau, \sigma}\left(k_{n}\right)$ as a manifestation of the time-reversal symmetry. Equation (23) can be recast in the vector form suitable for more complicated edge geometries:

$$
\begin{gathered}
\mathcal{M}=\cos \Lambda \tau_{0} \otimes \boldsymbol{\aleph} \otimes \boldsymbol{\Sigma}+\sin \Lambda \tau_{z} \otimes\left(\mathbf{n}_{B} \times \boldsymbol{\aleph}\right) \otimes \boldsymbol{\Sigma}, \\
\boldsymbol{\aleph}=\frac{1}{2}(\hat{\boldsymbol{e}}-i \hat{\boldsymbol{z}}) e^{i \zeta \hat{\boldsymbol{z}} \boldsymbol{\sigma}}+\frac{1}{2}(\hat{\boldsymbol{e}}+i \hat{\boldsymbol{z}}) e^{-i \zeta \hat{\boldsymbol{z}} \boldsymbol{\sigma}},
\end{gathered}
$$

where $\hat{\boldsymbol{e}}$ is a unit vector pointing along the edge, $\hat{z}$ is a unit vector normal to the plane, and the components of the vector $\aleph$ are operators acting in spin space.

The two spin subsystems separately form the quantum Hall states with the integer factors $N_{\uparrow}$ and $N_{\downarrow}$ obtained from equation (21) by substitution $\zeta \rightarrow \zeta \sigma$. However, there is no net Hall charge since $N_{\uparrow}+N_{\downarrow}=0$. Instead, from equation (21) we find the nonzero Hall spin, $S(\phi)=(\hbar / 2 e)\left(Q_{\uparrow}(\phi)-Q_{\downarrow}(\phi)\right)$, or, explicitly,

$$
S(\phi)=N \hbar\left(\phi-\frac{\operatorname{sgn} \phi}{2}\right), \quad N=\frac{N_{\uparrow}-N_{\downarrow}}{2} .
$$

Here $N(\zeta, \lambda)$ is given by equation (21). It is a singular function of the symmetry breaking parameters which determines the phase diagram of the system shown in Figure 2. The phases with $N=0$ correspond to ordinary zerogap semiconductor states with zero spin accumulation $\Delta S=S(1)-S(0)=0$, while the phases with $N= \pm 1$ can be identified as quantum spin Hall (QSH) states [20-23] 

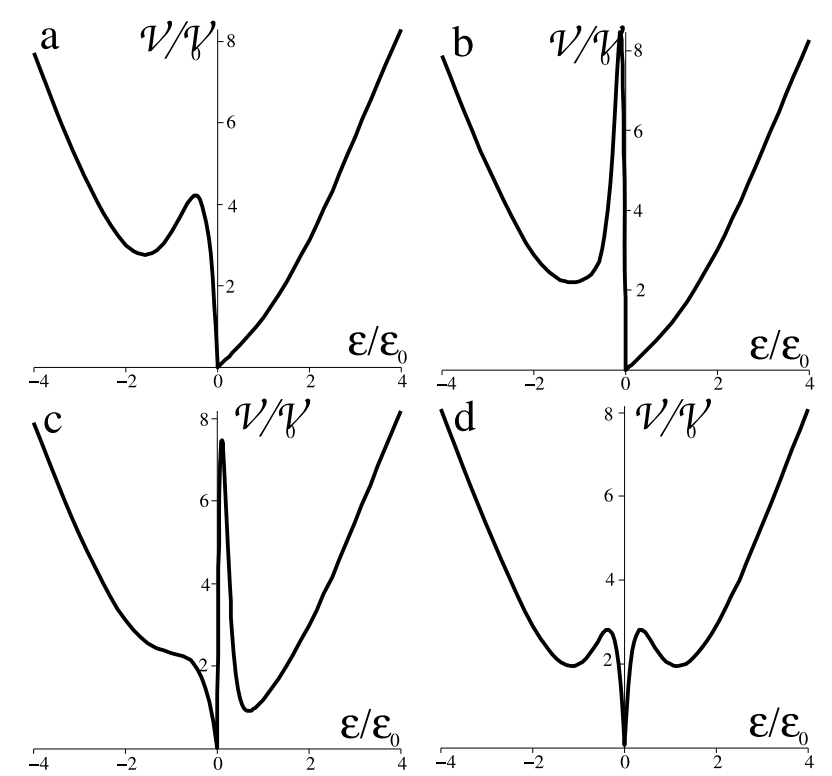

Fig. 3. LDOS at representative points of phase diagram in Figure 2: (a) $N=0$ phase: $\zeta=0, \lambda=0.4$, (b) close to transition from $N=0$ side: $\zeta=0.15, \lambda=0.25$, (c) close to transition from $N=-1$ side: $\zeta=0.3, \lambda=0.2$, and (d) $N=-1$ phase: $\zeta=0.3, \lambda=0$. We took the limit $L \rightarrow \infty$ in which only the $n=0$ terms in equations (17) and (18) contributed; $\varepsilon_{0}=\hbar v / 2 y$ and $\mathcal{V}_{0}=1 /$ hvy.

in which $\Delta S= \pm \hbar$. This identification is supported by the fact that the two valleys, $\tau= \pm 1$, provide a pair of spindependent edge states related by time-reversal symmetry (panel (c) in Fig. 2). Within such a pair the intervalley backscattering is forbidden unless there are time-reversal noninvariant interactions mixing the valleys and spin projections. In contrast, in the ordinary zero-gap semiconductor $(\zeta=0$ and $N=0)$ the edge states are spin degenerate (panel (a) in Fig. 2), and, therefore, spin-independent intervalley backscattering can lead to Anderson localization [12]. This is, for instance, the case for the edge states originally predicted by Fujita et al. [4] and their later generalizations (e.g. Ref. [16]). Also, unlike QSH topological insulators [20-23], in our case the edge states exist without any spin-orbit bulk energy gap, since they are supported by the nontrivial pseudospin structure of the zigzag boundary. The novelty of this type of edge states is most prominently manifested by the nonvanishing spin accumulation,

$$
S(\phi \rightarrow 0)=-N(\hbar / 2) \operatorname{sgn} \phi,
$$

resulting from the zero mode $n=0$ in equation (17).

Because of the gapless 2D bulk, transport measurements are hardly suitable for the characterization of the $N= \pm 1$ and $N=0$ phases. We propose a more robust method based on the tunneling spectroscopy of the LDOS. Figure 3 shows $\mathcal{V}(\epsilon, y)=\sum_{\sigma}\left[\mathcal{V}_{e}^{\sigma}(\epsilon, y)+\mathcal{V}_{b}^{\sigma}(\epsilon, y)\right]$, where $\mathcal{V}_{e, b}^{\sigma}(\epsilon, y)$ are given by equations (17) and (18) with $\zeta \rightarrow \zeta \sigma$. Panels (a) and (d) correspond to the $N=0$ and $N=-1$ phases, respectively, which differ by the symmetry of the energy dependence of $\mathcal{V}(\epsilon, y)$. The particle-hole asymmetric LDOS of the $N=0$ phase transforms into the symmetric LDOS of the $N=-1$ phase through a discontinuity (Figs. 3b and 3c) coming from zero-energy states existing on the critical lines of the diagram in Figure 2. The discontinuous phase transition implies that the $N= \pm 1$ states are topologically distinct from an ordinary zero-gap semiconductor with $N=0$.

\section{Conclusions}

Within the continuum model for zigzag terminated graphene, we have demonstrated the possibility of confinement mediated spin-orbit coupling. The system exhibits a phase transition into a quantum spin Hall state in the sense that it possesses a Kramers' doublet of spindependent edge states, accumulating an integer spin, albeit there is no true gap in the system's bulk as in the usual quantum spin Hall systems. We show that the local density of states can be used to distinguish the quantum spin Hall state from ordinary zero-gap semiconductor state of zigzag-terminated graphene. Our findings also imply that even in the absence of interaction-induced magnetism [4,28-30] zigzag graphene edges can be spin-active.

We thank H.U. Baranger, F. Guinea, M.I. Katsnelson and A.D. Mirlin for discussions. The work was supported by the Emmy-Noether Programme of the German Research Foundation (DFG).

\section{References}

1. K.S. Novoselov, A.K. Geim, S.V. Morozov, D. Jiang, M.I. Katsnelson, I.V. Grigorieva, S.V. Dubonos, A.A. Firsov, Nature (London) 438, 197 (2005)

2. Y. Zhang, Y.-W. Tan, H.L. Stormer, P. Kim, Nature (London) 438, 201 (2005)

3. L.A. Ponomarenko, F. Schedin, M.I. Katsnelson, R. Yang, E.H. Hill, K.S. Novoselov, A.K. Geim, Science 320, 356 (2008)

4. M. Fujita, K. Wakabayashi, K. Nakada, K. Kusakabe, J. Phys. Soc. Jpn 65, 1920 (1996); K. Nakada, M. Fujita, G. Dresselhaus, M.S. Dresselhaus, Phys. Rev. B 54, 17954 (1996)

5. K. Wakabayashi, M. Sigrist, Phys. Rev. Lett. 84, 3390 (2000)

6. Y. Kobayashi, K.I. Fukui, T. Enoki, K. Kusakabe, Y. Kaburagi, Phys. Rev. B 71, 193406 (2005)

7. Y. Niimi, T. Matsui, H. Kambara, K. Tagami, M. Tsukada, H. Fukuyama, Phys. Rev. B 73, 085421 (2006)

8. N.M.R. Peres, F. Guinea, A.H.C. Neto, Phys. Rev. B 73, 125411 (2006)

9. L. Brey, H.A. Fertig, Phys. Rev. B 73, 235411 (2006)

10. A. Rycerz, J. Tworzydlo, C.W.J. Beenakker, Nature Phys. 3, 172 (2007)

11. G. Tkachov, Phys. Rev. B 76, 235409 (2007)

12. K. Wakabayashi, Y. Takane, M. Sigrist, Phys. Rev. Lett. 99, 036601 (2007) 
13. H. Schomerus, Phys. Rev. B 76, 045433 (2007)

14. M. Hentschel, F. Guinea, Phys. Rev. B 76, 115407 (2007)

15. E.V. Castro, N.M.R. Peres, J.M.B. Lopes dos Santos, A.H.C. Neto, F. Guinea, Phys. Rev. Lett. 100, 026802 (2008); E.V. Castro, N.M.R. Peres, J.M.B. Lopes dos Santos, Europhys. Lett. 84, 17001 (2008)

16. A.R. Akhmerov, C.W.J. Beenakker, Phys. Rev. B 77, 085423 (2008)

17. G. Tkachov, Phys. Rev. B 79, 045429 (2009)

18. F.D.M. Haldane, Phys. Rev. Lett. 61, 2015 (1988)

19. G.W. Semenoff, Phys. Rev. Lett. 53, 2449 (1984)

20. C.L. Kane, E.J. Mele, Phys. Rev. Lett. 95, 226801 (2005)

21. L. Sheng, D.N. Sheng, C.S. Ting, F.D.M. Haldane, Phys. Rev. Lett. 95, 136602 (2005)

22. B.A. Bernevig, S.C. Zhang, Phys. Rev. Lett. 96, 106802 (2006)
23. M. König, S. Wiedmann, C. Brüne, A. Roth, H. Buhmann, L.W. Molenkamp, X.-L. Qi, S.-C. Zhang, Science 318, 766 (2007)

24. E. McCann, V.I. Fal'ko, J. Phys. Condens. Matter 16, 2371 (2004)

25. K. Sasaki, S. Murakami, R. Saito, Appl. Phys. Lett. 88, 113110 (2006)

26. R.B. Laughlin, Phys. Rev. B 23, 5632 (1981)

27. I. Žutić, J. Fabian, S. Das Sarma, Rev. Mod. Phys. 76, $323(2004)$

28. Y.-W. Son, M.L. Cohen, S.G. Louie, Nature 444, 347 (2006)

29. O.V. Yazyev, M.I. Katsnelson, Phys. Rev. Lett. 100, 047209 (2008)

30. M. Wimmer, I. Adagideli, S. Berber, D. Tomanek, K. Richter, Phys. Rev. Lett. 100, 177207 (2008) 